\title{
Temporal Lobe Epilepsy Masquerading as Tetany
}

Manish Parakh*, Anurag Singh and Sheesham Agarwal

Department of Pediatric Neurology, Dr S N Medical College, Jodhpur, India

${ }^{*}$ Corresponding author: Sheesham Agarwal, MBBS, 3rd year Resident Pediatrics Dr S N Medical College, Jodhpur (Raj), f-18/b-1, Bhannagar, Vaishali Nagar, Jaipur-302021, E-mail: agarwalsheesham@yahoo.com

Received date: June 01, 2015; Accepted date: June 22, 2015; Published date: June 26, 2015

Copyright: (2015 Parakh M. This is an open-access article distributed under the terms of the Creative Commons Attribution License, which permits unrestricted use, distribution, and reproduction in any medium, provided the original author and source are credited.

\section{Introduction}

Epilepsy is a common neurological disorder that affects about $1 \%$ of the general population. Temporal Lobe Epilepsy (TLE) is the one of the most common type of epilepsy [1].There are various signs and symptoms that can help physicians diagnose TLE. The most obvious sign is of course a seizure; however, there are several more subtle signs like memory loss, visual illusions and hallucinations, migraines, changes in heart rate, fear and anxiety, and pain abdomen [2].

Focal seizures presenting as tetany (carpopedal spasm) is a very rare manifestation of temporal lobe epilepsy. This presentation can be easily misdiagnosed as a manifestation due to hypocalcaemia and thus delay definite diagnosis and management of treatable epilepsy. This form of presentation has been previously reported in literature but continues to be a very rare presentation for both experienced Pediatric Neurologist and the Pediatric Internist. The following case report aims to add to the literature a rare presentation of treatable epilepsy.

\section{Case Report}

A 7 year old female child presented in the Pediatric Neurology clinic with history of having three episodes of tetanic carpopedal spasms in past 1 month. The first episode occurred when she was studying and suddenly felt numbness and tingling of both lower extremities followed by upper extremities. She then developed spasm of both wrists and posturing mimicking carpopedal spasm. She was taken to the nearest Emergency room where she was given injection calcium gluconate after which she improved \& was discharged on oral calcium. Serum calcium was not done because of non-availability of the same at this facility. Two days later she again had similar posturing of both hands along with mild feeling of discomfort of both feet. She was again taken to another emergency room where she improved within a few minutes. 12 hours after this admission she started complaining of pain in abdomen, pain in both hands and both feet along with fisting (posture as that of carpopedal spasm) of both hands. The posturing improved within 20-30 minutes of the admission. However she was hospitalised and treated with intravenous fluids and injection calcium gluconate. Serum Calcium, Random Blood Sugar, Serum Sodium, Potassium and EKG at the time of admission were within normal limits. Her .blood gas analysis also revealed normal $\mathrm{pH}$ normal $\mathrm{PaCO} 2, \mathrm{HCO} 3$ and base deficit. After this event she was referred to the Pediatric neurology clinic for further work up. In the Pediatric Neurology clinic a review history revealed that prior to the start of every event she always had a strange feeling in her lower extremity described as discomfort or numbness. She continued to feel the strange feeling in her both lower extremities especially distal leg and feet for around 10-30 minutes and this feeling was followed by a typical posturing of her both hands characterized by flexion at wrist joint and metacarpophalangeal joint and extension of interphalangeal joints with slight adduction of the thumb. She had no history of preceding vomiting, head trauma, ear discharge, involuntary defecation or urination during the episode, emotional changes or abdominal discomfort. She is a second issue born out of non consanguineous marriage with insignificant perinatal, natal and postnatal history, no family history of epilepsy and was not receiving any medications.

On examination she had anemia but no neurocutaneous stigmata, cyanosis, clubbing, edema or lymphadenopathy. On examination of the nervous system she was alert, well oriented to time, place, and person. Mental functions were normal, speech normal, no cranial nerve palsies. Tone \& power were normal. She was not having any abnormal movements. Other systems were normal.

Routine blood work up was within normal limits (Hemoglobin 9 $\mathrm{gm} / \mathrm{dl}$, Serum calcium $8.4 \mathrm{mg} / \mathrm{dl}$, ASAT $34 \mathrm{U} / \mathrm{l}$, ALAT $30 \mathrm{U} / \mathrm{l}$ ).The history of a preceding strange feeling in both her lower extremities followed by the typical posturing in both hands prompted us to keep seizure originating from the somatosensory area and then progressing to motor area as one of the possibilities,apart from carpopedal spam due to hypocalcemia or metabolic alkalosis. EEG was therefore performed which revealed presence of focal interictal epileptiform discharges in the form of 50-200 microvolt spike- slow wave complexes originating from T5 (Figure 1).

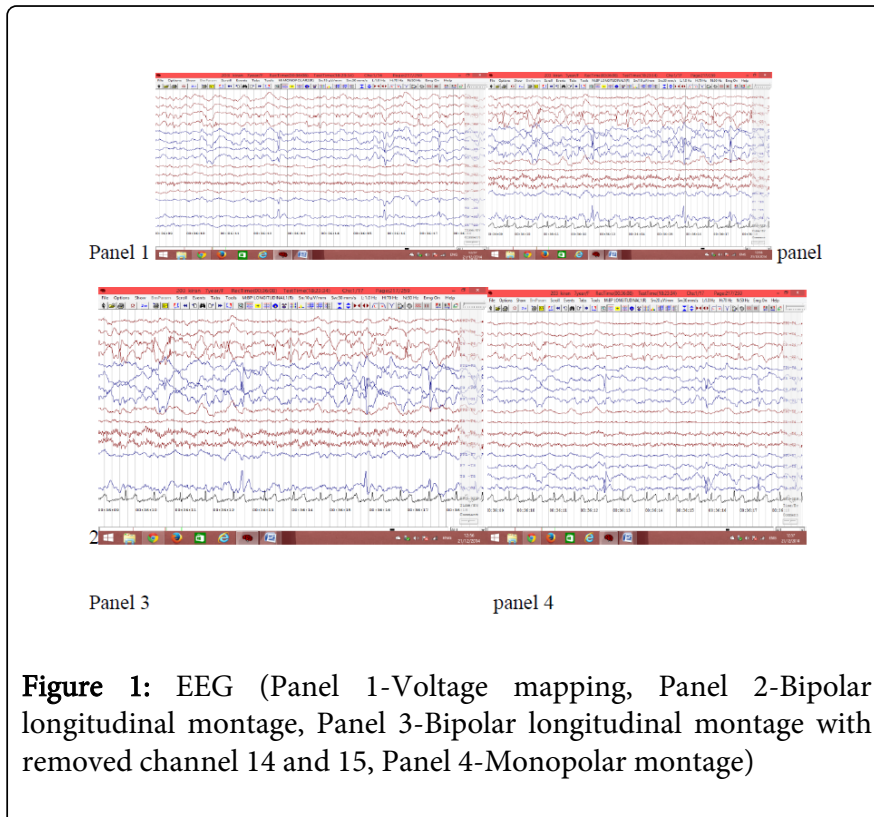

Patient was started with levetiracetam in the doses of $30 \mathrm{mg} / \mathrm{kg} /$ day. MRI brain revealed mild loss of volume and thinning of grey matter involving left hippocampal formation with prominence of adjacent temporal horn, however no significant T2/FLAIR hyperintense signals 
are noted from either of the hippocampus suggestive of mesial temporal sclerosis (Figure 2).

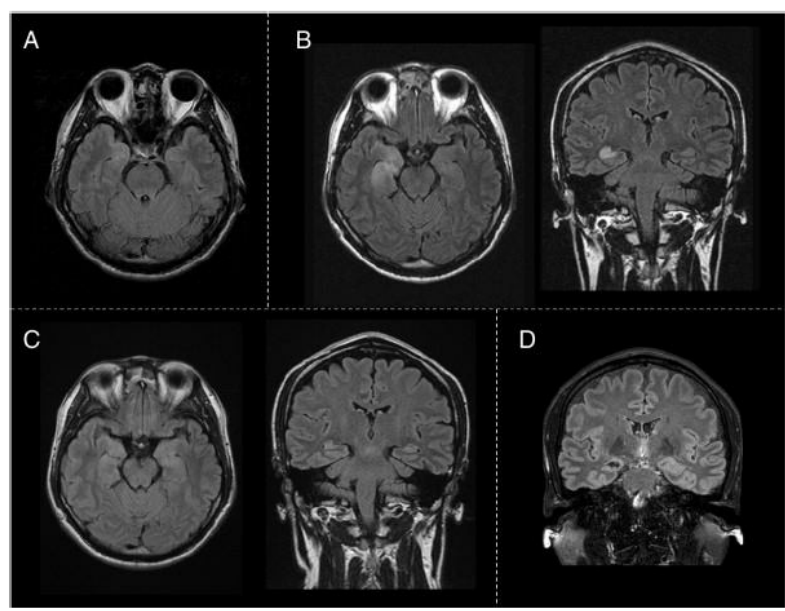

Figure 2: MRI images showing mesial temporal sclerosis

The patient is currently in her 5th month of follow up and has had no further episodes and is having a normal age appropriate neurodevelopment.

\section{Discussion}

Tetany is usually seen in children with hypocalcaemia and metabolic alkalosis. It may indicate enhanced neuromuscular activity and associated sensory disturbance [3]. The current patient presented to the treating clinician with a typical posturing suggestive of tetany and therefore was initially worked up for a cause of tetany. Her clinical picture did not suggest any setting for hypocalcemia or metabolic alkalosis and the work up also did not reveal any evidence for the same. On a review history in the Pediatric Neurology clinic a history of preceding strange feeling in both her lower extremities followed by the typical posturing prompted us to think of the abnormal motor event as a seizure. Subsequently, EEG findings and confirmation of the MRI findings demonstrating a structural lesion of left hippocampal area confirmed the diagnosis. Seizures originating from Temporal lobe may be preceded by an aura, can have typical stereotypic automatisms and have varied motor semiology including early non-forced head turn, late version, eye deviation, focal clonic jerking, fencing, tonic limb posturing, dystonic limb posturing, unilateral ictal paresis, postictal Todd's paresis [4]. Somatosensory aura occur very rarely as a part of Temporal lobe seizure semiology. Tonic and dystonic posturing in TLE may also be a part of automatisms [5]. In the current patient the dystonic posturing of hands mimicked tetany and therefore prompted the treating clinician to think of commoner etiologies of tetany (hypocalcemia and metabolic alkalosis). A review of history suggested that the strange feeling in the lower extremities was a part of preceding somatosensory aura and the dystonic posturing was actually a part of TLE seizure semiology. The current patient is an illustrative example of a rare presentation of TLE which is treatable and requires a detailed neurologic evaluation. However, it was mimicking a common clinical situation which posed diagnostic and management challenge to the Pediatric Internist. The case is therefore reported to increase awareness amongst the Pediatric Internist and the Pediatric Neurologist regarding tetany (dystonic posturing in this case) as one of the rare manifestation of a temporal lobe epilepsy amenable to specific treatment. Leviteracetam offers several advantages over traditional therapy, including twice daily dosing, a wide margin of safety with no requirements for serum drug concentration monitoring, and no interactions with other anticonvulsant. In addition, it appears to be well tolerated by most patients and have less adverse effects on cognitive function than traditional agents. It is widely used as a monotherapy in partial seizures and thus was used in this case.

\section{References}

1. Wang A, Peters TM, de Ribaupierre S, Mirsattari SM (2012) Functional magnetic resonance imaging for language mapping in temporal lobe epilepsy. Epilepsy Res Treat 2012: 198183.

2. Epilepsy.med.nyu.edu $>$ Information for Patients $>$ Types of Epilepsy

3. Ito N, Fukumoto S (2007) Symptoms and management of tetany. Clin Calcium 17: 1234-1239.

4. www.webmd.boots.com/a-to-z-guides/temporal-lobe-seizure

5. emedicine.medscape.com/article/1184509-overview 